\title{
Effects of interscalene brachial plexus block to intra-operative hemodynamics and postoperative pain for arthroscopic shoulder surgery
}

\author{
Hyun-Young Lee, Sang Hun Kim, Keum Yung So, and Dong Jun Kim \\ Department of Anesthesiology and Pain Medicine, Chosun University Hospital, Gwangju, Korea
}

Background: Although arthroscopic shoulder surgery is less invasive and painful than open shoulder surgery, it can often cause intra-operative hemodynamic instability and severe post-operative pain. This study was conducted to investigate the efficacy of the interscalene brachial plexus block (IBPB) on intra-operative hemodynamic changes and post-operative pain during arthroscopic shoulder surgery.

Methods: After institutional review board approval, 50 consecutive patients that had undergone arthroscopic shoulder surgery under general anesthesia were randomly assigned to one of two groups to evaluate intra-operative hemodynamic changes and post-operative pain control. Group 1 patients received an IBPB with $10 \mathrm{ml}$ of normal saline guided by a nerve stimulator before induction, and Group 2 patients received $10 \mathrm{ml}$ of $0.5 \%$ ropivacaine hydrochloride with the same technique. The heart rate and systolic and diastolic blood pressures were recorded before the incision and 1, 3, 5, 10, and 20 minutes after the incision. Pre-operative and post-operative pain was evaluated with a visual analog scale 1, 3, 6, 12, and 24 hours after surgery. The patients were given tramadol as a rescue medication option. The total volume of tramadol that was injected was also evaluated over the same intervals. Results: Group 2 showed significantly lower systolic and diastolic blood pressures and heart rates intra-operatively compared to Group $1(\mathrm{P}<0.05)$. The visual analog scale pain scores, except at 24 hours after surgery, were significantly lower in Group $2(\mathrm{P}<0.05)$. The total tramadol consumption significantly reduced in Group $2(\mathrm{P}<0.05)$. Conclusions: IBPB effectively controlled the hemodynamic changes that occurred during arthroscopic shoulder surgery as well as post-operative pain. (Korean J Anesthesiol 2012; 62: 30-34)

Key Words: Arthroscopy, Brachial plexus, Hemodynamics, Nerve block, Pain, Shoulder.

Received: January 31, 2011. Revised: 1st, April 19, 2011; 2nd, June 21, 2011; 3rd, August 25, 2011. Accepted: August 25, 2011.

Corresponding author: Sang Hun Kim, M.D., Ph.D., Department of Anesthesiology and Pain Medicine, Chosun University Hospital, 588, Seasuk-dong, Dong-gu, Gwangju 501-717, Korea. Tel: 82-62-220 3223, Fax: 82-62-223-2333, E-mail: ksh3223@chosun.ac.kr

(c) This is an open-access article distributed under the terms of the Creative Commons Attribution Non-Commercial License (http:// creativecommons.org/licenses/by-nc/3.0/), which permits unrestricted non-commercial use, distribution, and reproduction in any medium, provided the original work is properly cited. 


\section{Introduction}

Although arthroscopic shoulder surgery has the advantages of decreased scarring, faster recovery due to decreased overall pain and infection, and a shorter admission period compared with open shoulder surgery, it often causes intra-operative hemodynamic instability and severe post-operative pain [1-3]. Therefore, it is important to have an effective method of minimizing intra-operative hemodynamic changes and reducing post-operative pain. The interscalene brachial plexus block (IBPB) is commonly used for these purposes as it can effectively regulate acute post-operative pain that occurs approximately 8-10 hours after the surgery, and has a high success and low complication rate [4-8]. This study was conducted to investigate the effect of IBPB on hemodynamic changes before anesthesia administration during arthroscopic shoulder surgery and severe post-operative pain.

\section{Materials and Methods}

This study was conducted on 50 patients aged 18-60 years who were scheduled to undergo arthroscopic shoulder surgery due to rotator cuff tear, and whose condition corresponded to physical status I and II of the American Society of Anesthesiologists (ASA). After the approval of the institutional review board, the study was conducted after obtaining informed consent of the study purpose, risks, and complications from each patient. Those who did not give their consent and had a coagulopathy, a cardiothoracic and vascular disease, or a medical record of chronic pain, or were sensitive to local anesthetics, or taking drugs that may affect their blood pressure or heart rate were excluded from the study.

All the subjects were randomly assigned to the following two groups using a random number table: a group that was subjected to IBPB with $10 \mathrm{ml}$ of normal saline before the surgery (Group 1, $\mathrm{n}=25$ ) and a group that was subjected to IBPB with $10 \mathrm{ml}$ of $0.5 \%$ ropivacaine hydrochloride before the surgery (Group 2, $\mathrm{n}=25$ ). Before the treatment, intramuscular 0.05 $\mathrm{mg} / \mathrm{kg}$ of midazolam was injected $30 \mathrm{~min}$ before the induction of anesthesia, and their vital signs were measured using an ECG, a pulse oximeter, and a blood pressure measuring device immediately after they arrived at the operation room. After their vital signs were stabilized, they were asked to turn their faces towards the direction opposite to the treatment side in a supine position. The patient's skin was punctured using a 50 mm 22 G needle (Stimuplex ${ }^{\circledR}$ A, B. Braun, Germany) with the assistance of a nerve stimulator (PAJUNK ${ }^{\circledR}$ Medizintechnologie, Geisingen, Germany) to identify the exact needle location. If the triceps, biceps, or deltoid or pectoralis major muscle twitched when an electric stimulus of $1 \mathrm{~Hz}$ and $1 \mathrm{~mA}$ was applied, the electric stimulus was gradually reduced to $0.5 \mathrm{~mA}$, and the point at which the muscle contraction was maintained was set as the blocking point. While observing the blood injection via aspiration, $1 \mathrm{ml}$ of $0.5 \%$ ropivacaine hydrochloride was slowly injected. If the response loss was confirmed, the needle was placed at the correct blocking location. Subsequently, the remaining $9 \mathrm{ml}$ of $0.5 \%$ ropivacaine hydrochloride was injected. If no response to a $0.3 \mathrm{~mA}$ or lower stimulus was found, nerve blocking was considered to have been achieved. The IBPB was administered by an experienced anesthesiologist, and the arthroscopic shoulder surgery was also conducted by one surgeon.

As for the induction of anesthesia, $2 \mathrm{mg} / \mathrm{kg}$ of propofol and $0.9 \mathrm{mg} / \mathrm{kg}$ of rocuronium were administered to both groups, followed by tracheal intubation. Anesthesia was maintained using a 1.5 minimal alveolar concentration (MAC) of sevoflurane with $2 \mathrm{~L} / \mathrm{min}$ of $\mathrm{O}_{2}$ and $2 \mathrm{~L} / \mathrm{min}$ of $\mathrm{N}_{2} \mathrm{O}$. For continuous blood pressure monitoring, a $22 \mathrm{G}$ arterial catheter was installed in the radial artery under local anesthesia after a modified Allen's test.

The systolic arterial pressure (SAP), diastolic arterial pressure (DAP), and heart rate (HR) that were measured after the vital signs were stabilized were recorded (Tb), and a skin incision was made one minute later. The vital signs were recorded 1 (T1), 3 (T3), 5 (T5), 10 (T10), 15 (T15), and $20 \mathrm{~min}$ (T20) after the start of the surgery. If SAP $\geq 190 \mathrm{mmHg}$ or DAP $\geq 120 \mathrm{mmHg}$, $15 \mu \mathrm{g} / \mathrm{kg}$ of nicardipine was administered. On the other hand, if $\mathrm{SAP} \leq 80 \mathrm{mmHg}, 5 \mathrm{mg}$ of ephedrine or $50 \mu \mathrm{g}$ of phenylephrine was administered repeatedly. If $\mathrm{HR} \leq 50$ beats $/ \mathrm{min}, 0.5 \mathrm{mg}$ of atropine was administered. The patents that had undergone drug treatment were excluded from the study.

The patients were transferred to the recovery room after the extubation. Upon passive exercise of shoulder abduction and adduction, the pain score was recorded using the Visual Analog Scale (VAS) 1 (T1 h), 3 (T3 h), 6 (T6 h), 12 (T12 h), and 24 hours (T24 h) after the surgery.

The standard value of VAS (Tb) was set as the score during passive exercise before the surgery. When patients with a pain score of 5 in the recovery room and the patient room wanted analgesics, $1 \mathrm{mg} / \mathrm{kg}$ of tramadol was repeatedly administered to them and recorded.

Except for the patient number, sex ratio (\%), and frequency of use of additional analgesics, all the measured values were denoted with their mean \pm standard deviation. A statistical analysis was conducted using SPSS 17.0 for Windows (SPSS, Inc., Chicargo, IL). A Chi-squared test or a Fisher's exact test was conducted for the sex and frequency of using analgesics, and a Student's unpaired t-test was conducted for the patient's height, age, and weight. A repeated measures ANOVA test was conducted for the systolic and diastolic blood pressures, 
heart rate, and VAS scores, and a Mann-Whitney U-test was conducted for back-testing. A P value $<0.05$ was considered statistically significant.

\section{Results}

A total of 50 patients were registered in this study without withdrawal. No significant difference in age, height, weight, and sex was found between the two groups (Table 1).

Table 1. Demographic Data

\begin{tabular}{lcc}
\hline & $\begin{array}{c}\text { Group 1 } \\
(\mathrm{n}=25)\end{array}$ & $\begin{array}{c}\text { Group 2 } \\
(\mathrm{n}=25)\end{array}$ \\
\hline ASA physical status (I/II) & $17 / 8$ & $19 / 6$ \\
Age (yr) & $46.8 \pm 10.2$ & $52.5 \pm 15.5$ \\
Sex (M/F) & $14 / 6$ & $11 / 9$ \\
Weight (kg) & $69.7 \pm 8.3$ & $64.9 \pm 7.0$ \\
Height (cm) & $169.5 \pm 7.4$ & $166.8 \pm 5.8$ \\
Preoperative VAS (mm) & $39.1 \pm 3.1$ & $39.1 \pm 3.2$
\end{tabular}

Data are presented as the mean \pm SD and numbers of patient. Group 1 patients received an interscalenebrachial plexus block with normal saline $10 \mathrm{ml}$ guided by nerve stimulator before induction, and Group 2 patients received the same technique with $0.5 \%$ ropivacaine $10 \mathrm{ml}$. ASA: American Society of Anesthesiologists, VAS: visual analogue scale.

A
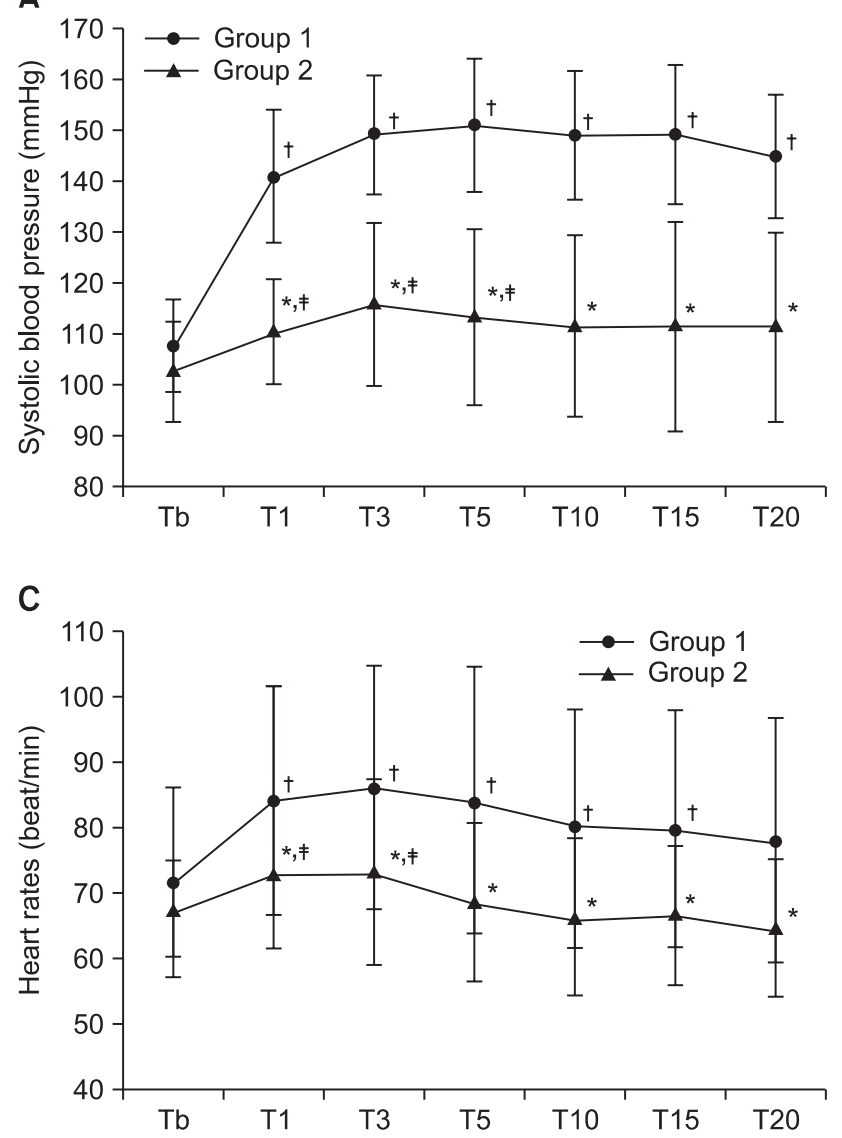

The systolic and diastolic blood pressures and heart rates changed more significantly in Group 1, which underwent IBPB using normal saline after skin incision, than in Group 2, which underwent IBPB using $0.5 \%$ ropivacaine ( $P<0.05$, Fig. 1 ). In Group 1, significantly elevated systolic and diastolic blood pressures were measured at all time points, unlike before the skin incision. The heart rate significantly increased at all the time points, except at 20 min after the skin incision $(\mathrm{P}<0.05)$. In Group 2, elevated systolic and diastolic blood pressures occurred only $5 \mathrm{~min}$ after the skin incision. No significant change in heart rate was seen, except 3 min after the skin incision $(\mathrm{P}<$ 0.05 , Fig. 1). No hypertension, hypotension, and bradycardia that required drug treatment was found in both groups.

No significant difference in the VAS score was found between the two groups when the scores were assessed in the patient room before the surgery. The VAS score by time was significantly higher in Group 2 than in Group 1. No significant difference was found 24 hours after the surgery $(\mathrm{P}<0.05$, Fig. 2$)$.

The frequency of the additional use of analgesics significantly increased in Group 1 until 12 hours after the surgery $(\mathrm{P}<0.05$, Fig. 3), but no significant difference was found between the two groups in 24 hours after the surgery.

B

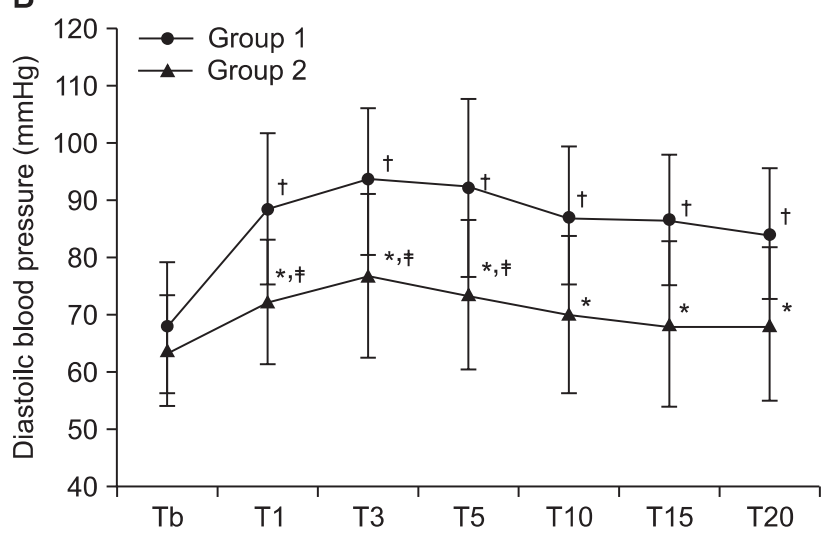

Fig. 1. Changes in systolic arterial blood pressure (A), diastolic arterial blood pressure (B) and heart rates (C). Data are expressed as mean \pm SD. There were significant differences between the two groups after skin incision in systolic arterial blood pressure, diastolic arterial blood pressure and heart rates. Group $1(n=25)$ received an interscalene brachial plexus block with normal saline $10 \mathrm{ml}$ guided by nerve stimulator before induction, and Group 2 $(\mathrm{n}=25)$ received the same technique with $0.5 \%$ ropivacaine $10 \mathrm{ml}$. Tb: before incision, T1: 1 minute after incision, T3: 3 minutes after incision, T5: 5 minutes after incision, T10: 10 minutes after incision, T15: 15 minutes after incision, T20: 20 minutes after incision. ${ }^{*} \mathrm{P}<$ 0.05 compared with Group $1,{ }^{\dagger} \mathrm{P}<0.05$ compared with $\mathrm{Tb}$ in group 1 , ${ }^{\ddagger} \mathrm{P}<0.05$ compared with $\mathrm{Tb}$ in group 2 . 


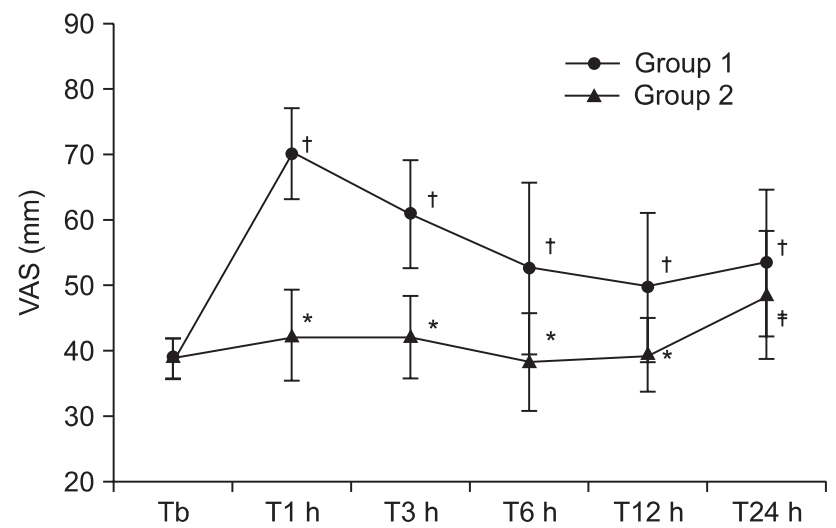

Fig. 2. Visual analog scale (VAS) at passive exercise before surgery and after 1, 3, 6, 12 and $24 \mathrm{~h}$ postoperatively. There were significant differences in Group 2 compared with Group 1 except at 24 hours postoperatively. Group $1(n=25)$ received an interscalene brachial plexus block with normal saline $10 \mathrm{ml}$ guided by nerve stimulator before induction, and Group $2(n=25)$ received the same technique with $0.5 \%$ ropivacaine $10 \mathrm{ml}$. Tb: preoperative, $\mathrm{T} 1 \mathrm{~h}: 1$ hour postoperatively, T3 h: 3 hours postoperatively, T6 h: 6 hours postoperatively, T12 h: 12 hours postoperatively, T24 h: 24 hours postoperatively. ${ }^{*} \mathrm{P}<0.05$ compared with Group $1,{ }^{\dagger} \mathrm{P}<0.05$ compared with $\mathrm{Tb}$ in group $1,{ }^{\ddagger} \mathrm{P}<0.05$ compared with $\mathrm{Tb}$ in group 2 .

\section{Discussion}

In this study, intra-operative hemodynamic stability was maintained in Group 2, in which the patients underwent IBPB using $\mathbf{0 . 5 \%}$ ropivacaine before arthroscopic shoulder surgery. In addition, significant pain reduction and a reduced additional requirement for analgesics were shown in Group 2 for the 12 hours after the surgery, unlike in Group 1, in which patients underwent IBPB using a saline solution.

In arthroscopic shoulder surgery, minimization of hemodynamic changes and pain control after the surgery are very important. IBPB, which controls unstable hemodynamic changes and post-operative pain during arthroscopic shoulder surgery, has the advantages of intra-operative bleeding reduction, excellent muscle relaxation, reduced risks and complications due to general anesthesia, reduced requirement for additional analgesics after the surgery, and a shorter hospitalization duration [9]. In addition, due to its high success rate, low complication rate, and faster effect, it is an effective method as the sole anesthesia for the upper extremities and during arthroscopic shoulder surgery $[7,8,10]$. Singelyn et al. [10] reported that IBPB showed a greater analgesic effect than the suprascapuler nerve block or intra-articular injection for 24 hours after the surgery. Furthermore, they showed that IBPB reduced the requirement for narcotic analgesics after the surgery and minimized the risk of post-operative nausea and vomiting. IBPB complications have been reported from time to time. Whitaker et al. [11] reported that severe hypotension

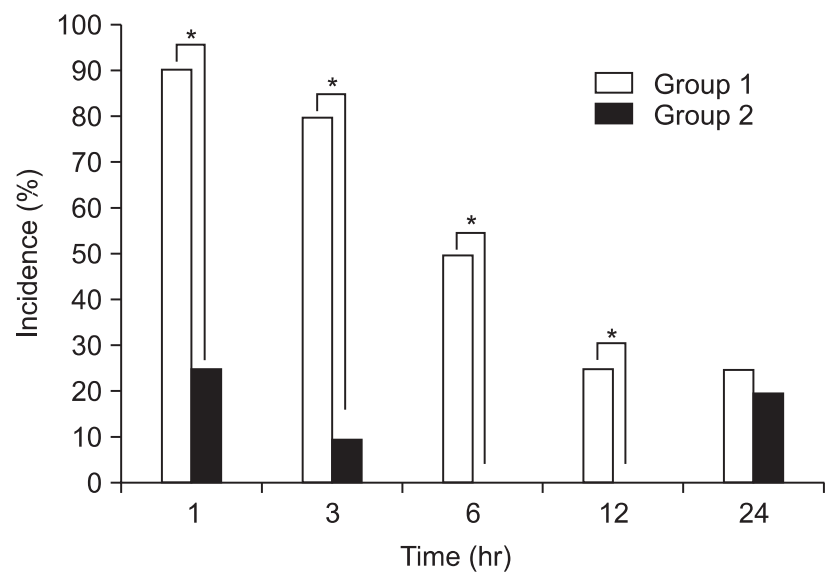

Fig. 3. The incidence of additional analgesic requirements after 1 , $3,6,12$ and 24 h postoperatively. There were significant increases in group 1 except at 24 hours after surgery. Group $1(n=25)$ received an interscalene brachial plexus block with normal saline $10 \mathrm{ml}$ guided by nerve stimulator before induction, and group $2(n=25)$ received a same technique with $0.5 \%$ ropivacaine $10 \mathrm{ml}$. $* \mathrm{P}<0.05$ compared with Group 1.

occurred during arthroscopic shoulder surgery after IBPB. They added that local anesthetics injected during IBPB caused a neuroaxial block. In this study, the post-operative pain and the frequency of the additional use of analgesics more significantly decreased in the group that underwent IBPB using $0.5 \%$ ropivacaine than in the group that underwent IBPB using a saline solution. However, no significant changes in the VAS scores and the frequency of the additional use of analgesics were found after postoperative 12 hours. This result was similar to that of the study of Conroy et al. [5], in which one-time BPB effectively controlled the pain for a mean of 10.5 hours. This is likely to have been caused by the short-term efficacy of the local anesthesia that was used.

As no reports of hemodynamic instability such as increased blood pressure and heart rate during arthroscopic shoulder surgery have been suggested in previous studies, a few causes were hypothesized before this study. First, hemodynamic instability could be caused by surgical manipulation during anesthesia. Morrison et al. [12] reported that the difference between systolic blood pressure and shoulder joint pressure should be maintained at $49 \mathrm{mmHg}$ or lower to secure the surgery field in their study on the relationship between shoulder joint pressure, blood pressure, and surgery field in arthroscopic shoulder surgery. They also reported that a low blood pressure should be maintained during the surgery for lower injection pressure of the irrigation fluid and to minimize the risk of fluid extravasation to the subcutaneous tissue. However, there are no reports to date showing intra-operative hemodynamic instability caused by increased shoulder joint pressure due to increased injection pressure of the irrigation fluid. In this study, 
frequent changes in the injection pressure of the irrigation fluid were required due to the poor surgery field caused by the increased blood flow in the joint, which was caused by significantly increased blood pressure and heart rate in the group that underwent IBPB using a normal saline solution. In the group that underwent IBPB using $0.5 \%$ ropivacaine, the surgery and anesthetic management were conducted with a stable hemodynamic status and without increasing the injection pressure in the irrigation fluid. Although injection pressure in the irrigation fluid was observed in this study, it was not recorded. Thus, it cannot be concluded whether the injection pressure in the irrigation fluid was a direct cause of hemodynamic changes. It can be inferred, however, that the hemodynamic changes reduced due to blocking of the pain caused by surgical treatment, as IBPB using ropivacaine blocked the nerve that innervated the shoulder joint. Second, the changes could have occurred due to the systemic absorption of epinephrine that was included in the irrigation fluid. Jensen et al. [13] reported that $0.33 \mathrm{mg} / \mathrm{L}$ of epinephrine effectively improved the bleeding inside the joint and the surgery field without causing adverse cardiovascular events in arthroscopic surgery. The severe hemodynamic changes that occurred due to epinephrine during arthroscopic shoulder surgery were mainly attributable to accidents due to carelessness. Cho et al. [3] reported that ventricular tachycardia suddenly occurred due to the systemic absorption of epinephrine contained in the irrigation fluid during arthroscopic shoulder surgery. They also reported that unstable hemodynamic changes could be prevented if epinephrine was mixed well with the irrigation fluid. Karns [14] reported that ventricular tachycardia occurred due to the intraosseous infusion of the irrigation fluid that contained epinephrine at a $1: 100,000$ ratio, which was caused by humeral cortex injury during the trochar insertion. In this study, $0.33 \mathrm{mg} / \mathrm{L}$ of epinephrine was used, and none of the aforementioned severe cardiovascular complications was observed in both groups. Although significant changes in blood pressure and heart rate were observed in the group that underwent IBPB using a saline solution, this is likely to have been due to the elevated blood pressure caused by surgical manipulation rather than by the systemic absorption of epinephrine.

This study had the following limitations. First, we did not record the injection pressure changes in the irrigation fluid which was mentioned as a cause of hemodynamic changes. Second, we did not evaluate the systemic absorption of $0.33 \mathrm{mg} /$ L of epinephrine. Third, only a one-time IBPB was conducted without sufficient consideration of the pain-persistent duration after arthroscopic shoulder surgery. Thus, the effect of IBPB was not assessed 24 hours after the surgery. We suggest that further investigation on hemodynamic changes due to the injection pressure in the irrigation fluid, the blood epinephrine level and the effect of continuous IBPB on pain control is required.

In summary, the hemodynamic changes that occurred during arthroscopic shoulder surgery were caused by the inappropriate blocking of pain transmission that occurred due to surgical manipulation. IBPB, which showed stable hemodynamic changes during arthroscopic shoulder surgery, is an effective method to control early pain 12 hours after surgery.

\section{References}

1. Chung F, Ritchie E, Su J. Postoperative pain in ambulatory surgery. Anesth Analg 1997; 85: 808-16.

2. Rawal N, Hylander J, Nydahl PA, Olofsson I, Gupta A. Survey of postoperative analgesia following ambulatory surgery. Acta Anaesthesiol Scand 1997; 41: 1017-22.

3. Cho SH, Yi JW, Kwack YH, Park SW, Kim MK, Rhee YG. Ventricular tachycardia during arthroscopic shoulder surgery: a report of two cases. Arch Orthop Trauma Surg 2010; 130: 353-6.

4. Wu CL, Rouse LM, Chen JM, Miller RJ. Comparison of postoperative pain in patients receiving interscalene block or general anesthesia for shoulder surgery. Orthopedics 2002; 25: 45-8.

5. Conroy BP, Gray BC, Fischer RB, Del Campo LJ, Kenter K. Interscalene block for elective shoulder surgery. Orthopedics 2003; 26: 501-3.

6. Chao D, Young S, Cawley P. Postoperative pain management for arthroscopic shoulder surgery: interscalene block versus patientcontrolled infusion of $0.25 \%$ bupivicaine. Am J Orthop (Belle Mead NJ) 2006; 35: 231-4.

7. Bishop JY, Sprague M, Gelber J, Krol M, Rosenblatt MA, Gladstone JN, et al. Interscalene regional anesthesia for arthroscopic shoulder surgery: a safe and effective technique. J Shoulder Elbow Surg 2006; 15: 567-70.

8. Faryniarz D, Morelli C, Coleman S, Holmes T, Allen A, Altchek D, et al. Interscalene block anesthesia at an ambulatory surgery center performing predominantly regional anesthesia: a prospective study of one hundred thirty-three patients undergoing shoulder surgery. J Shoulder Elbow Surg 2006; 15: 686-90.

9. Brown AR, Weiss R, Greenberg C, Flatow EL, Bigliani LU. Interscalene block for shoulder arthroscopy: comparison with general anesthesia. Arthroscopy 1993; 9: 295-300.

10. Singelyn FJ, Lhotel L, Fabre B. Pain relief after arthroscopic shoulder surgery: a comparison of intraarticular analgesia, suprascapular nerve block, and interscalene brachial plexus block. Anesth Analg 2004; 99: 589-592.

11. Whitaker EE, Edelman AL, Wilckens JH, Richman JM. Severe hypotension after interscalene block for outpatient shoulder surgery: a case report. J Clin Anesth 2010; 22: 132-4.

12. Morrison DS, Schaefer RK, Friedman RL. The relationship between subacromial space pressure, blood pressure, and visual clarity during arthroscopic subacromial decompression. Arthroscopy 1995; 11: 557-60.

13. Jensen KH, Werther K, Stryger V, Schultz K, Falkenberg B. Arthroscopic shoulder surgery with epinephrine saline irrigation. Arthroscopy 2001; 17: 578-81.

14. Karns JL. Epinephrine-induced potentially lethal arrhythmia during arthroscopic shoulder surgery: a case report. AANA J 1999; 67: 419-21. 